\title{
Influence of drying methods on the quality of Orthosiphon stamineus extract
}

\author{
Cher Haan Lau ${ }^{a}$, Lee Suan Chua ${ }^{\text {a,b, }}{ }^{*}$ \\ a Institute of Bioproduct Development, Universiti Teknologi Malaysia, 81310 UTM Johor Bahru, Johor, Malaysia \\ ${ }^{b}$ Department of Bioprocess and Polymer Engineering, Faculty of Chemical and Energy Engineering, Universiti Teknologi Malaysia, 81310 UTM \\ Johor Bahru, Johor, Malaysia \\ * Corresponding author: Ischua@ibd.utm.my
}

\section{Article history}

Submitted 20 December 2017

Revised 14 February 2018

Accepted 28 February 2018

Published Online 30 April 2018

\section{Graphical abstract}

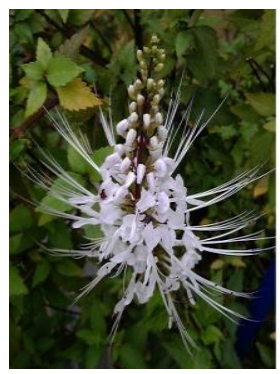

\begin{abstract}
The interest in herbal products is tremendously increasing in recent years, probably due to their health promoting benefits. Drying is one of the important processes in herbal extract preparation. Therefore, this study evaluated the effects of drying methods on the quality of Orthosiphon stamineus (OS) extract. The OS extract was concentrated by a centrifugal concentrating system operated at three different modes (Infrared (IR), IR-Heat and Heat), vacuum evaporator (VE) and oven dryer. The phytochemical profiles of the dried extracts were analysed by LC-MS/MS. The oven-dried OS extract showed the highest yield (26.2\%), followed by heat-dried, IR-heat-dried, IRdried and VE-dried. The VE-dried extract showed the highest reduction of extract quality in term of its phytochemical content, particularly for the major compounds such as caffeic acid, eupatorin, eupatorin derivative, myricetin, rosmarinic acid, rutin and sinensetin. The drying kinetic for IR, heat and IR-heat methods were fitted well into Page model with $R^{2}>0.99$. This research concluded that the selection of drying method is important because it will affect the phytochemical profile of the plant extract significantly.
\end{abstract}

Keywords: Orthosiphon stamineus, LC-MS/MS, herbal extract, rosmarinic acid, eupatorin

\section{INTRODUCTION}

Orthosiphon stamineus is a member of Orthsiphon genus in the plant family Lamiaceae. It has been widely used as traditional supplement for health improvement and illness treatment. $O$. stamineus also posseses several pharmacological activities such as antioxidant, anti-inflamatory, anti-diabetic and anti-bacterial (Ameer et al., 2012). Previous studies reported the isolation of various active compounds from $O$. stamineus including caffeic acid, rosmarinic acid, sinensetin, eupatorin and orthosiphol A-Z.

Drying is an important process in herbal processing industries, particularly for drying raw plant material and plant extract. Dried herbal extract has better stability, consistent quality than liquid extract (Conway, 2007). The stability of herbal extract is significantly affected by physical properties (moisture content, $\mathrm{pH}$ and particle size), chemical properties (phytochemical content and solvent used for extraction) and storage conditions (temperature, light and humidity) (Thakur et al., 2011). Plant extract are usually dried using the methods of rotary evaporation under vacuum, oven drying, infrared drying and heat drying. Modern drying techniques including freeze-drying and spray-drying which are widely applied in the dehydration of herbal extract in large scale (Gallo et al., 2015).

In this study, the effects of drying methods on the phytochemical content of crude extract from $O$. stamineus were investigated. Different drying techniques have been used by previous investigators, but no conclusive comparison on the content of plant metabolites has been carried out till to date. The drying kinetic was also determined to compare the efficiency of the drying methods in term of moisture transfer.

\section{EXPERIMENTAL}

\section{Materials}

Analytical grade of ethanol $(96 \%)$ was purchased from Fisher Scientific Co. (Fair Lawn, NJ). HPLC grade of methanol, acetonitrile and formic acid were purchased from Merck (Darmstadt, Germany). The standard chemicals of caffeic acid, eupatorin, euparorin derivative, myricetin, rosmarinic acid and rutin were purchased from Sigma-Aldrich (St. Louis, MO, USA) Sinensetin was supplied by ChromaDex (Irvine, CA, USA). The dried plant material was purchased from Fidea Resources (Selangor, Malaysia).

\section{Preparation of Orthosiphon stamineus extract}

The plant material $(10 \mathrm{~g})$ was extracted with $70 \%$ ethanol $(200$ $\mathrm{mL})$ at the ratio of $1: 20(\mathrm{w} / \mathrm{v})$ for 2 hours under reflux condition (Chua and Lau, 2016). The solution was then filtered by filter paper.

\section{Drying procedures}

The drying processes such as oven drying, infrared (IR), IR-heat and heat assisted techniques were carried out using $1.5 \mathrm{~mL}$ extracted solution in $2 \mathrm{~mL}$ centrifugal tubes in triplcate. Only vacuum evaporation (VE) was performed in larger volume, $10 \mathrm{~mL}$.

A laboratory scale forced convection oven (Daihan LabTech LDO-250F, Korea) was used to dry plant extract at $50^{\circ} \mathrm{C}$ until completely dried.

A rotary evaporator (Heidolph, Laborota 4003, Germany) equipped with reflux cooler (ROTACOOL) and vacuum pump (ROTAVAC vario control) was used to perrform VE (200 mbar) at $50^{\circ} \mathrm{C}$. 
A centrifugal concentrator equipped with cold trap system (Micro-Cenvac NB 503CIR, N-BIOTEK Co. Ltd., Korea) with horsepower of $200 \mathrm{~W}$ and frequency of $50 / 60 \mathrm{~Hz}$, was used to dry at $50^{\circ} \mathrm{C}$. The concentrator was operated with three different modes (IR, IR-Heat and Heat).

\section{Kinetic study of drying process}

The kinetic profiles of infrared (IR) drying, IR-heat drying and heat drying were investigated based on the moisture content of plant extract. The moisture loss was recorded at 10 minutes interval by weighing the sample. The moisture content $(\mathrm{M})$ and moisture ratio (MR) of samples were calculated by Eq. (1) and (2), respectively ( $\mathrm{Li}$ et al., 2017).

$$
\begin{aligned}
& M=\frac{\text { Mass of water }(\mathrm{g})}{\text { Mass of dry extract }(\mathrm{g})} \\
& M R=\frac{M_{t}-M_{e}}{M_{0}-M_{e}}
\end{aligned}
$$

where $M_{t}, M_{0}, M_{e}$ are the moisture content at time $t$, initial and equilibrium, respectively. The kinetic models (Table 1) were used to describe the drying kinetic. The goodness-of-fit of the proposed models was compared in terms of coefficient of determination $\left(\mathrm{R}^{2}\right)$, root mean square error (RMSE) and Chi-square $\left(\chi^{2}\right)$. The parameter of the models was estimated by Excel Solver (Microsoft Office Professional Plus 2013).

Table 1 Kinetic equations for drying models.

\begin{tabular}{lll}
\hline \multicolumn{1}{c}{ Model } & \multicolumn{1}{c}{ Equation } & \multicolumn{1}{c}{ Reference } \\
\hline Henderson-Pabis & $M R=a \exp (-k t)$ & (Panceri et al., 2013) \\
Page & $M R=\exp \left(-k t^{c}\right)$ & (Darvishi et al., \\
& 2014) \\
Lewis & $M R=\exp (-k t)$ & (Bensebia and Allia, \\
Logarithmic & $M R=a \exp (-k t)+c$ & 2015) \\
\hline
\end{tabular}

\section{LC-MS/MS analysis of dried extract}

The identification and quantification of bioactive compounds were performed by a liquid chromatography system equipped with a diode array detector ((Dionex, Ultimate 3000, Thermo Scientific; MA, USA) and a C18 reversed phase column $(2.1 \times 100 \mathrm{~mm}, 2.5 \mu \mathrm{m}$, XSelect High Strength Silica, Waters; Milford, MA). The separation was performed using mobile phases consisted of $0.1 \%$ formic acid in water (A) and $0.1 \%$ formic acid in methanol (B) with a gradient elution at the flow rate of $200 \mu \mathrm{L} / \mathrm{min}: 0-5 \mathrm{~min}, 35 \% \mathrm{~B} ; 5-15 \mathrm{~min}, 35$ $70 \% \mathrm{~B} ; 15-20 \mathrm{~min}, 70-90 \% \mathrm{~B} ; 20-25 \mathrm{~min}, 90 \% \mathrm{~B} ; 25-26 \mathrm{~min}, 90$ $35 \% \mathrm{~B} ; 26-30 \mathrm{~min}, 35 \% \mathrm{~B}$. All samples were filtered prior to injection.

The fragments of target metabolites were used to verify the presence of the compounds using standard chemicals. The QTOF mass spectrometer was used for the small metabolite screening from $\mathrm{m} / \mathrm{z}$ 100-1000. A single Information Dependent Acquisition (IDA) method was created to acquire both TOF MS and two dependent runs of product ion scan with rolling collision energy. Nitrogen gas was used for nebulizing (40 psi) and curtain gas (20 psi). Collision gas was set at 3, the accumulation time was $1 \mathrm{~s}$ for TOF MS and $2 \mathrm{~s}$ for each product ion scan. The voltage of ion spray was $-4500 \mathrm{~V}$ for negative ion mode. The declustering potential was $40 \mathrm{~V}$ and the focusing potential was set at $300 \mathrm{~V}$.

\section{RESULTS AND DISCUSSION}

\section{Effect of drying methods on phytochemical content}

Drying is an important process to improve the stability and quality consistency of herbal extract. However, most of bioactive compounds are heat sensitive. Prolong exposure of heat might lead to the degradation of active ingredients in herbal products (WHO, 2017). Fig 1 shows the relationship of drying methods and the phytochemical content. The figure clearly shows that the phytochemical content reduced significantly after drying, particularly for the key phytochemicals such as caffeic acid, eupatorin, eupatorin derivative, myricetin, rosmarinic acid, rutin and sinensetin.

Overall, the oven-dried extract showed the lowest phytochemical loss, followed by heat-dried, IR-dried, IR-heat-dried and VE-dried. VE caused the most significant loss in phytochemical content because of direct exposure to heat during flask rotation. This acelerated VE drying process $(30 \mathrm{~min}$ ) by exploring larger surface area of solution. Since the herbal solution was dried at low vapour pressure, the boiling point of solvent was lowered, and thus the heat transfer rate could be increased (Michailidis and Krokida, 2014).

Oven or conventional drying could provide uniform heating environment with circulation of hot air throughout the oven (Mauer and Bradley Jr, 2017). However, this method is time and energy consuming compared to other methods. Although oven drying showed the minimum loss of phytochemicals, this method took the longest duration, almost 24 hours to completely dryness.

The drying modes of centrifugal concentrator did not show any significant trend in phytochemical degradation (Fig. 1). The degradation was in a compound dependent manner. Rutin was found to be more IR sensitive than sinensetin. Caffeic acid and rosmarinic acid were more resistant to heat contributed by drying. Flavonoids (eupatorin, myricetin, sinensetin) and flavonoid derivatives (eupatorin derivative and rutin) showed larger loss than phenolic acids (caffeic acid and rosmarinic acid).

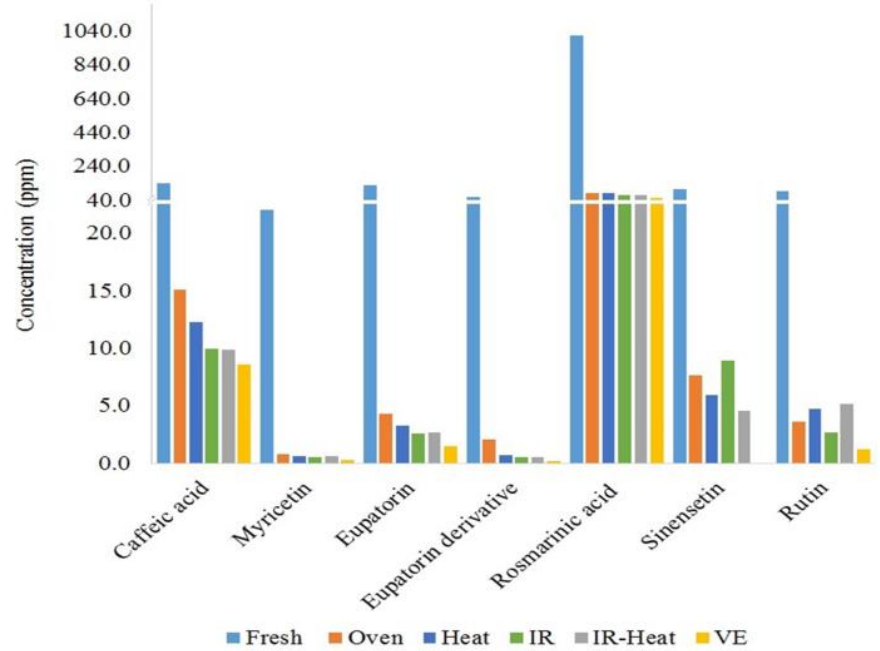

(a)

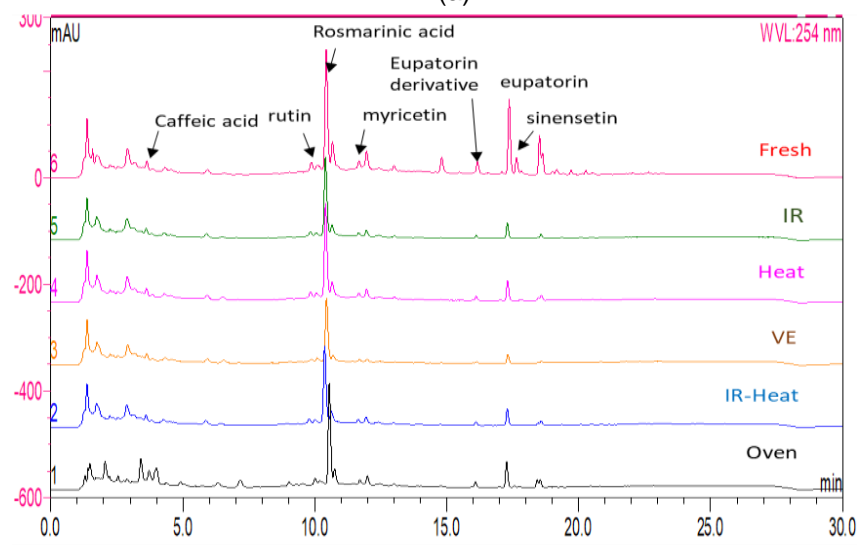

(b)

Fig. 1 (a) Concentration of few detected phytochemicals in $O$. stamineus extracts prepared by the drying methods of oven, heat, infrared (IR), infra-heat (IR-heat) and vacuum evaporation (VE) as shown in (b) their chromatograms at $254 \mathrm{~nm}$. 
IR drying involves heat penetration by IR radiation in sample matrix. The absorption energy is strongly depended on the wavelength of the IR radiation. Most of the biological material absorb radiative energy in the far-IR (FIR) region (Sandu, 1986). Based on Wien's displacement law (Krishnamurthy et al., 2008), the IR drying occurred at $50^{\circ} \mathrm{C}$ was in the FIR range $(8.97 \mu \mathrm{m})$. The inner temperature of sample was higher than surrounding air (Michailidis and Krokida, 2014). According to Krishnamurthy et al. (2008), IR heating might change the physical, chemical, and functional properties of compounds. Therefore, IR drying seemed degrading more phytochemicals than other techniques (Fig. 1). The low phytochemical content of IR drying could be explained by its heating mechanism in which sample absorbs IR radiation without energy loss. Thus, the absorbed energy led rapid conversion of liquid to dried solid (Senevirathne et al., 2010). Hence, IR drying method exhibited shorter dehydration duration (180 $\mathrm{min})$ than IR-heat $(220 \mathrm{~min})$ and heat $(240 \mathrm{~min})$ modes. However, sinensetin was found to be higher content in IR dried extract, rutin was also higher in IR-heat dried extract than oven dried extract. The observation indicates that the absorption of IR energy was also strongly influenced by the structure of the compounds.

Fig. 2 compares the drying methods with their extraction yields of $O$. stamineus. Oven-dried extract has the highest extraction yield, but VE-dried extract was the lowest yield, which is in good agreement with phytochemical content.

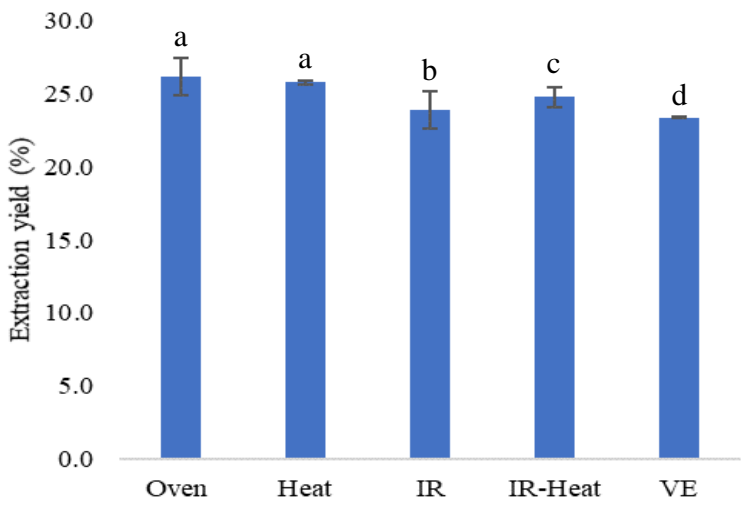

Fig. 2 Effect of drying methods on extraction yield, different small letter on top of the bar indicates significant different at $p<0.05$ using oneway analysis of variance.

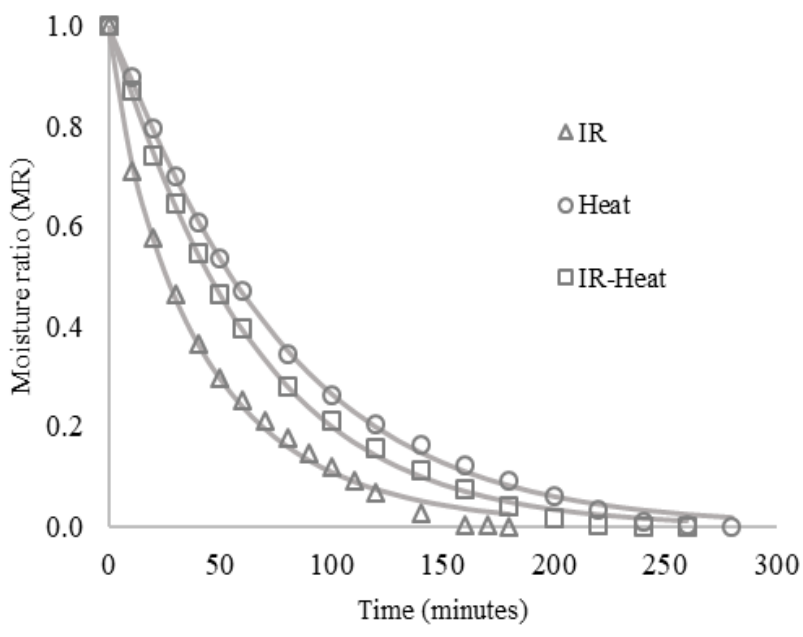

Fig. 3 Experimental and theoretical moisture ratios predicted by Page model for different concentrating methods.

\section{Kinetic profile of drying process}

Drying kinetic is widely applied to express the reduction rate of moisture content (Michailidis and Krokida, 2014). In the present study, the drying kinetic of IR, IR-heat and heat methods were investigated because they did not have significant trend in phytochemical loss for comparison. Possibly, this could be due to the type of phytochemicals selected in this study.

The drying kinetic was monitored based on the loss of moisture content. The kinetic data was fitted with the proposed models, namely Henderson-Pabis, Page, Lewis and logarithmic. The curve fitting results were presented in Table 2. The results show that Page model was the most suitable model to describe the drying kinetic of IR $\left(M R=\exp \left(-0.042 t^{0.863}\right)\right.$, heat $\left(M R=\exp \left(-0.009 t^{1.090}\right)\right.$ and IR-heat ( $\mathrm{MR}=\exp \left(-0.012 \mathrm{t}^{1.066}\right)$ drying methods with the highest $\mathrm{R}^{2}$, but the lowest RMSE and $\chi^{2}$.

The kinetic profiles of the drying processes are illustrated in Fig. 3. The IR assisted method shows higher drying rate than IR-heat and heat assisted methods, especially for the first hour of drying. The observation was in line with the duration taken by the processes during sample drying.

Table 2 Statistical results and estimated parameters of drying models for different concentrating methods.

\begin{tabular}{|c|c|c|c|c|c|c|c|c|c|c|c|c|}
\hline \multirow{2}{*}{ Model } & \multicolumn{4}{|l|}{ IR } & \multicolumn{4}{|l|}{ Heat } & \multicolumn{4}{|c|}{ IR-Heat } \\
\hline & $\mathbf{R}^{2}$ & RMSE & $x^{2}$ & Parameters & $\mathbf{R}^{2}$ & RMSE & $x^{2}$ & Parameters & $\mathbf{R}^{2}$ & RMSE & $x^{2}$ & Parameter \\
\hline Henderson-Pabis & 0.993 & 0.023 & 0.080 & $\begin{array}{l}\mathrm{a}=0.950 \\
\mathrm{k}=0.023\end{array}$ & 0.998 & 0.014 & 0.060 & $\begin{array}{l}a=1.025 \\
k=0.013\end{array}$ & 0.998 & 0.014 & 0.084 & $\begin{array}{l}a=1.017 \\
k=0.016\end{array}$ \\
\hline Page & 0.996 & 0.017 & 0.101 & $\begin{array}{l}\mathrm{k}=0.042 \\
\mathrm{c}=0.863\end{array}$ & 0.999 & 0.011 & 0.058 & $\begin{array}{l}\mathrm{k}=0.009 \\
\mathrm{c}=1.090\end{array}$ & 0.999 & 0.010 & 0.060 & $\begin{array}{l}\mathrm{k}=0.012 \\
\mathrm{c}=1.066\end{array}$ \\
\hline Lewis & 0.990 & 0.028 & 0.092 & $\begin{array}{l}\mathrm{k}=0.024 \\
\mathrm{a}=0.949\end{array}$ & 0.997 & 0.018 & 0.099 & $\begin{array}{l}\mathrm{k}=0.013 \\
\mathrm{a}=1.026\end{array}$ & 0.998 & 0.015 & 0.091 & $\begin{array}{l}\mathrm{k}=0.016 \\
\mathrm{a}=1.017\end{array}$ \\
\hline Logarithmic & 0.993 & 0.028 & 0.082 & $\begin{array}{l}\mathrm{k}=0.023 \\
\mathrm{~b}=0.001\end{array}$ & 0.998 & 0.016 & 0.086 & $\begin{array}{l}\mathrm{k}=0.013 \\
\mathrm{~b}=0\end{array}$ & 0.998 & 0.014 & 0.084 & $\begin{array}{l}\mathrm{k}=0.016 \\
\mathrm{~b}=0\end{array}$ \\
\hline
\end{tabular}

\section{CONCLUSION}

This study revealed that different drying methods could affect the quality of herbal extract because of phytochemical degradation. Oven drying at low temperature $\left(50^{\circ} \mathrm{C}\right)$ could preserve the quality of extract, but longer drying time is required which may not be economic in large scale drying. IR assisted technique could be the method of choice to dry $O$. stamineus extract. However, some phytochemicals might be degraded at the minimum level compared to other techniques.

\section{ACKNOWLEDGEMENT}

The authors would like to thank the Ministry of Higher Education, Malaysia (HiCoE 4J263) for the financial support.

\section{REFERENCES}

Ameer, O. Z., Salman, I. M., Asmawi, M. Z., Ibraheem, Z. O., and Yam, M. F. (2012). Orthosiphon stamineus: Traditional Uses, Phytochemistry, Pharmacology, and Toxicology. Journal of Medicinal Food, 15(8), 678690 . 
Bensebia, O., and Allia, K. (2015). Drying and extraction kinetics of rosemary leaves: Experiments and modeling. Journal of Essential Oil Bearing Plants, 18(1), 99-111.

Conway, B. R. (2007). Solid dosage forms. In Gad, S. C. (Ed.) Pharmaceutical Manufacturing Handbook: Production \& Processes (pp. 233-265). New Jersey: John Wiley \& Sons, Inc.

Chua, L. S., and Lau, C. H. (2016). Reflux extraction and column chromatography for rosmarinic acid-rich fraction from Orthosiphon stamineus. The Natural Products Journal, 7(1), 30-36.

Darvishi, H., Asl, A. R., Asghari, A., Azadbakht, M., Najafi, G., and Khodaei, J. (2014). Study of the drying kinetics of pepper. Journal of the Saudi Society of Agricultural Sciences, 13(2), 130-138.

Gallo, L., Ramírez-Rigo, M. V., Piña, J., and Bucalá, V. (2015). A comparative study of spray-dried medicinal plant aqueous extracts. Drying performance and product quality. Chemical Engineering Research and Design, 104(Supplement C), 681-694.

Krishnamurthy, K., Khurana, H. K., Soojin, J., Irudayaraj, J., and Demirci, A. (2008). Infrared heating in food processing: An overview. Comprehensive Reviews in Food Science and Food Safety, 7(1), 2-13.

Li, Y.-h., Qi, Y.-r., Wu, Z.-f., Wang, Y.-q., Wang, X.-c., Wang, F., Yang, M. (2017). Comparative study of microwave-vacuum and vacuum drying on the drying characteristics, dissolution, physicochemical properties, and antioxidant capacity of Scutellaria extract powder. Powder Technology, $317,430-437$.
Mauer, L. J., and Bradley Jr, R. L. (2017). Moisture and Total Solids Analysis. In Nielsen, S. S. (Ed.) Food Analysis (pp. 257-286). USA: Springer.

Michailidis, P. A., and Krokida, M. K. (2014). Drying and dehydration processes in food preservation and processing. In Bhattacharya, S. (Ed.) Conventional and Advanced Food Processing Technologies (pp. 1-32). New Jersey: John Wiley \& Sons, Ltd.

Panceri, C. P., Gomes, T. M., De Gois, J. S., Borges, D. L. G., and BordignonLuiz, M. T. (2013). Effect of dehydration process on mineral content, phenolic compounds and antioxidant activity of Cabernet Sauvignon and Merlot grapes. Food Research International, 54(2), 1343-1350.

Sandu, C. (1986). Infrared Radiative Drying in Food Engineering: A Process Analysis. Biotechnology Progress, 2(3), 109-119.

Senevirathne, M., Kim, S.-H., Kim, Y.-D., Oh, C.-K., Oh, M.-C., Ahn, C.-B., et al. (2010). Effect of far-infrared radiation drying of citrus press-cakes on free radical scavenging and antioxidant activities. Journal of Food Engineering, 97(2), 168-176.

Thakur, L., Ghodasra, U., Patel, N., and Dabhi, M. (2011). Novel approaches for stability improvement in natural medicines. Pharmacognosy Reviews, 5(9), 48-54.

WHO (2017). WHO guidelines on good herbal processing practices (GHPP) for herbal medicines. Geneva: World Health Organization. 